\title{
EGF/EGFR upregulates and cooperates with Netrin-4 to protect glioblastoma cells from DNA damage-induced senescence
}

$\mathrm{Li} \mathrm{Li}^{1 \dagger}$, Yulun Huang ${ }^{2 \dagger}$, Yuge Gao ${ }^{1 \dagger}$, Tengfei Shi ${ }^{1}$, Yunyun $\mathrm{Xu}^{3}$, Huini $\mathrm{Li}^{4}$, Marko Hyytiäinen ${ }^{4}$, Jorma Keski-Oja ${ }^{4}$, Qiuying Jiang ${ }^{1^{*}}$, Yizhou $\mathrm{Hu}^{4,6^{*}}$ (D) and Zhimin $\mathrm{Du}^{5^{*}}$

\begin{abstract}
Background: Glioblastoma multiforme (GBM) is the most malignant central nervous system tumor. Alkylating agent, temozolomide (TMZ), is currently the first-line chemotherapeutic agent for GBM. However, the sensitivity of GBM cells to TMZ is affected by many factors. And, several clinic trials, including co-administration of TMZ with other drugs, have failed in successful treatment of GBM. We have previously reported that Netrin-4 (NTN4), a laminin-like axon guidance protein, plays a protective role in GBM cell senescence upon TMZ-triggered DNA damage. However, the master regulator of NTN4 needs further elucidation. Epidermal growth factor/Epidermal growth factor receptor (EGF/EGFR) can modulate the expression of various extracellular matrix related molecules, and prevent DNA damage in GBM cells. In this study, we investigated the relationship between EGF/EGFR signaling and NTN4, and explored their effect on therapeutic efficacy in GBM cells upon TMZ treatment.
\end{abstract}

Methods: Co-expression analysis were performed by using the RNA sequencing data from NIH 934 cell lines and from single cell RNA sequencing data of GBM tumor. The co-expressing genes were used for GO enrichment and signaling pathway enrichment. mRNA expression of the target genes were quantified by $q P C R$, and cell senescence were investigated by Senescence-Associated Beta-Galactosidase Staining. Protein phosphorylation were observed and analyzed by immunoblotting. The RNA sequencing data and clinical information of TMZ treated patients were extracted from TCGA-glioblastoma project, and then used for Kaplan-Meier survival analysis.

Results: Analysis of RNA sequencing data revealed a potential co-expression relationship between NTN4 and EGFR. GO enrichment of EGFR-correlated genes indicated that EGFR regulates GBM cells in a manner similar to that in central nervous system development and neural cell differentiation. Pathway analysis suggested that EGFR and its related genes contribute to cell adhesion, extracellular matrix (ECM) organization and caspase related signaling. We also show that EGF stimulates NTN4 expression in GBM cells and cooperates with NTN4 to attenuate GBM cell senescence induced by DNA damage, possibly via AKT and ERK. Clinical analysis showed that co-expression of EGFR and NTN4 significantly predicts poor survival in TMZ-treated GBM patients.

(Continued on next page)

\footnotetext{
*Correspondence: jiangqiuying@medmail.com.cn; yizhou.hu@ki.se; dzm1956@126.com

${ }^{+} \mathrm{Li}$ Li, Yulun Huang and Yuge Gao contributed equally to this work. 'Department of Oncology, the Second Clinical College, Harbin Medical University, Harbin, People's Republic of China

${ }^{4}$ Departments of Virology and Pathology, Faculty of Medicine, the Haartman

Institute, Translational Cancer Biology Research Program and Helsinki

University Hospital, University of Helsinki, Helsinki, Finland

${ }^{5}$ Department of pharmacy, the Second Clinical College, Harbin Medical

University, Harbin, People's Republic of China

Full list of author information is available at the end of the article
}

(c) The Author(s). 2018 Open Access This article is distributed under the terms of the Creative Commons Attribution 4.0 International License (http://creativecommons.org/licenses/by/4.0/), which permits unrestricted use, distribution, and reproduction in any medium, provided you give appropriate credit to the original author(s) and the source, provide a link to the Creative Commons license, and indicate if changes were made. The Creative Commons Public Domain Dedication waiver (http://creativecommons.org/publicdomain/zero/1.0/) applies to the data made available in this article, unless otherwise stated. 
(Continued from previous page)

Conclusions: This study indicates that EGF/EGFR regulates and cooperates with NTN4 in DNA damage resistance in GBM. Therefore, our findings provide a potential therapeutic target for GBM.

Keywords: Epidermal growth factor, Netrin-4, Glioblastoma, Cell senescence

\section{Background}

Glioblastoma multiforme (GBM) is the most common malignant tumor of the central nervous system $[1,2]$. Even with treatment, the median survival time of glioblastoma patients following diagnosis is $12 \sim 15$ months $[3,4]$. Alkylating agent, temozolomide (TMZ), is currently the first-line chemotherapeutic agent for the treatment of glioblastoma. The therapeutic action of TMZ is exerted by triggering DNA damage, which induces GBM cell apoptosis and senescence [5]. However, even with adjuvant TMZ chemotherapy, the 2-year overall survival rate of GBM patients only reaches $~ 27 \%$ [6-8]. The therapeutic efficacy of TMZ depends on self-repair of DNA damage [6], the rescue effect from RTK signaling [9-11], and cell remodeling in the microenvironment [12].

Our previous study showed that Netrin-4 (NTN4) protects glioblastoma cells from TMZ-induced senescence through rescuing the phosphorylation of AKT and ERK [13]. NTN4 was originally identified as a secreted laminin-like axon guidance protein in neuronal development $[14,15]$. In addition to the central nervous system, NTN4 is broadly expressed in non-neural cells, and regulates tissue development and tumor progression by modulating various cellular functions [16-23]. In glioblastoma, NTN4 is highly expressed at the tumor invading edge [24] and promotes glioblastoma cell proliferation via ITGB4-Akt signaling [25]. Although the expression of netrin-associated receptors including ITGA6 and ITGB4, is regulated by various growth factors, especially by Epidermal growth factor/Epidermal growth factor receptor EGF/EGFR [26-29], the upstream regulators of NTN4 expression and of GBM cell senescence remain largely unknown.

EGF is a growth factor that stimulates cell growth, proliferation, and differentiation [30]. EGF binds to EGFR family receptors with high affinity. This stimulates ligand-induced dimerization, activating the intrinsic protein-tyrosine kinase activity of the receptor. The tyrosine kinase activity, in turn, initiates signal transduction cascades that results in a variety of biochemical changes within the cell: a rise in intracellular calcium levels, increased glycolysis and protein synthesis, and increases in the expression of certain genes including the gene for EGFR, that lead to the regulation of various biological functions [31, 32]. EGF/EGFR signaling can protect cells from DNA damage through different downstream signaling pathways [33-35]. However, clinical trials of EGF/EGFR inhibitors failed to show any therapeutic efficacy, even in combination with $\operatorname{TMZ}[36,37]$. Therefore, upon induction of DNA damage in GBM cells, co-inhibition of RTK signaling and the extracellular matrix remodeling, may provide a new strategy for GBM treatment. Here, using experimental and bioinformatic analyses, we describe EGF/EGFR upregulation of NTN4 expression and EGF/EGFR cooperation with NTN4 to attenuate GBM cell senescence induced by DNA damage.

\section{Methods}

Immunoblot analysis, immunofluorescence, transfection of cells, total RNA extraction, reverse transcription, and real-time reverse transcription-PCR were performed as described [13, 25]. Senescence-associated beta-galactosidase staining and bioinformatic analysis were performed as below.

\section{Cell lines and reagents}

U251MG cells (Health Science Research Resources Bank, Osaka, Japan) were cultured according to the supplier's instructions. Primary GBM cells were isolated according to previous description [38, 39], and then cultured in in Dulbecco's modified essential culture medium (DMEM) supplied with $10 \%$ heat inactivated fetal calf serum (FCS) (Gibco, USA), $100 \mathrm{IU} / \mathrm{ml}$ penicillin, $50 \mathrm{mg} / \mathrm{ml}$ streptomycin and $1 \%$ L-glutamine.. In this study, all experiments have obtained patients' consent and been approved by the Ethic Committee for Harbin Medical University. The following primary antibodies and recombinant protein were used: anti-NTN4 from R\&D systems; anti-phospho-p44/43MAPK (ERK1/2, Thr202/Tyr204), anti-phospho-mammalian target of rapamycin (mTOR; Ser2448), anti-phospho-SRC(Ser416), and anti-phospho-AKT (Ser473) from Cell Signaling Tech. (Danvers, MA, USA); anti- $\beta$-tubulin from Santa Cruz Biotechnology (Santa Cruz, CA; recombinant NTN4 from R\&D Systems (Minneapolis, MN, USA).

\section{$\mathrm{H}_{2} \mathrm{O}_{2}$ and EGF treatment}

$\mathrm{H}_{2} \mathrm{O}_{2}$ (Merck KGaA, Germany) treatment was performed on $>90 \%$ confluent cells $\left(\sim 1 \times 10^{5}\right.$ cells per well in 6 -well plates) to avoid variability of $\mathrm{H}_{2} \mathrm{O}_{2}$ toxicity as described in [40]. Cells were incubated in $2 \mathrm{ml} /$ well culture medium containing $150 \mu \mathrm{M} \mathrm{H}_{2} \mathrm{O}_{2}$ for $48 \mathrm{~h}$ at $37^{\circ} \mathrm{C}$ in a $5 \% \mathrm{CO}_{2}$ atmosphere, and then split to 5000 cells per well in 24-well plates in fresh DMEM. 
EGF (Sigma E9644) treatment was performed at a concentration of $50 \mathrm{ng} / \mathrm{ml}$, and was added to growing cells in $0.05 \%$ serum DMEM in 24-well plates every 2 days. Subsequently, the cells were gently washed twice with PBS, and then cultured under 10\% serum fresh DMEM for further experiments.

\section{Transfection of cells}

Cells were cultured in six-well plates to 50 to $80 \%$ confluence and transfected using $2 \mu \mathrm{g}$ plasmid DNA and $6 \mu \mathrm{l}$ FuGENE 6 transfection reagent for each transfection according to the manufacturer's instructions (Boehringer Mannheim, Mannheim, Germany). Stably transfected cells

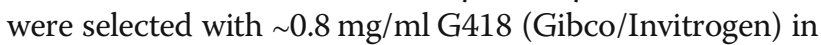
complete medium. The expression levels of each indicated gene in transfected cells were analyzed by Q-RT-PCR.

\section{Senescence-associated Beta-galactosidase staining (SA $\beta$-gal staining)}

Cells were cultured in 24-well plates at densities of 3000 5000 cells per well for the indicated times. Subsequently, the cells were washed once with PBS and then fixed in PBS containing $0.5 \%$ glutaraldehyde at room temperature for $20 \mathrm{~min}$. After two PBS washes, the cells were incubated with SA $\beta$-gal substrate solution $(1 \mathrm{mg} /$ $\mathrm{ml} \mathrm{X}$-gal, $40 \mathrm{mM}$ citric acid-sodium phosphate buffer, $\mathrm{pH}$ 6.0, containing $5 \mathrm{mM}$ potassium ferricyanide, $5 \mathrm{mM}$ potassium ferrocyanide, $150 \mathrm{mM} \mathrm{NaCl}$, and $2 \mathrm{mM}$

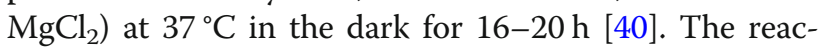
tion was terminated by removing SA $\beta$-gal substrate solution and washing twice with PBS. The cells were stored in $70 \%$ glycerol at $4{ }^{\circ} \mathrm{C}$. The cells were photographed using an Axiovert 200 inverted epifluorescence microscope (Carl Zeiss). The ratio of blue-stained cells was analyzed with Image (National Institutes of Health, Bethesda, MD).

\section{Bioinformatic analysis}

Co-expression analysis was performed using Spearman's rank correlation coefficient between the expression of EGFR and all other genes, in the dimension of all single cells. Distance Correlation analysis of gene expression was described previously [41-43]. Briefly, for each gene, pairwise Euclidean distances were generated in the dimension of all cells. After scaling the values by mean, the co-variance of EGFR and other genes was calculated upon the distance matrix between EGFR and all other genes. GO enrichment was assessed using Python Package jdrudolph/goenrich with modification on the enrichment of GO bioprocess, and the pathway enrichment was analyzed by Python Package PyPathway. Lifelines Package was used for Kaplan-Meier survival analysis. 3D axes plots and Violin plots were generated by Matplotlib and Seaborn.

\section{Statistical analysis}

All numerical values represent the mean $\pm \mathrm{SE}$ or $\mathrm{SD}$. Statistical significance was determined with the nonparametric Mann-Whitney U test.

\section{Results}

EGF stimulates Netrin-4 expression in U251MG cells

ITGA6, ITGB4 are regulated by different growth factors [20, 27, 44-47]. Therefore, as a potential ligand of laminin integrins, NTN4, is possibly expressed in response to stimulation by growth factors. By analyzing RNA sequencing data of NIH 934 human cell lines [48], we explored the co-expression relationships among NTN4, ITGA6, ITGB4, EGFR, and ErBB2 in these cell lines. We found that expression of NTN4 significantly correlated with EGFR (Fig. 1a). Meanwhile, we confirmed that the expression of EGFR is tightly linked to that of ITGA6 and ITGB4. These findings are in line with our previous observation that NTN4/ITGB4 protect GBM cells from TMZ-induced cell senescence, and indicate that NTN4 expression is possibly monitored by the EGF signaling pathway. To further understand the downstream pathway and biological functions of EGF signaling in glioblastoma, we extracted single-cell RNA sequencing (scRNAseq) data of grade IV glioblastoma [49]. After overlapping the results from Spearman correlation coefficient and Distance (pDist) Correlation analyses, we obtained a list of 191 genes that significantly correlate with EGFR, including NTN4 (SI Table I). Using these 191 genes, we further enriched for GO bioprocess and signaling pathways underlying EGFR signaling (SI Table II). Cell adhesions, ECM organization and Caspase-related signaling pathways were the top effectors of EGFR expression (Fig. 1b). Nervous system development and glial cell differentiation were the top GO bio-processes of EGFR-related genes (Fig. 1c). To validate this observation, U251MG GBM cells and two primary GBM cell lines, GBM-14042 and GBM-112D cells, were treated with EGF for $24 \mathrm{~h}, 48 \mathrm{~h}$, and $96 \mathrm{~h}$. U251MG express the mRNA of both EGFR and NTN4 at decent level, but does not express MGMT [13, 50], and the similar expression profile were detected in both GBM-14042 and GBM-112D cells by qRT-PCR (Fig. 1d). In addition, both of GBM-14042 and GBM-112D cells do not express MGMT (negative data, not shown here). Furthermore, we observed that NTN4 expression were significantly increased upon EGF stimulation at both mRNA level (Fig. 1e) and protein level (Fig. 1f).

The GO function and signaling pathway enrichment revealed that EGFR might contribute to cell differentiation, central nervous system development, ECM re-organization, and netrin/DCC related caspase activation. Meanwhile, 


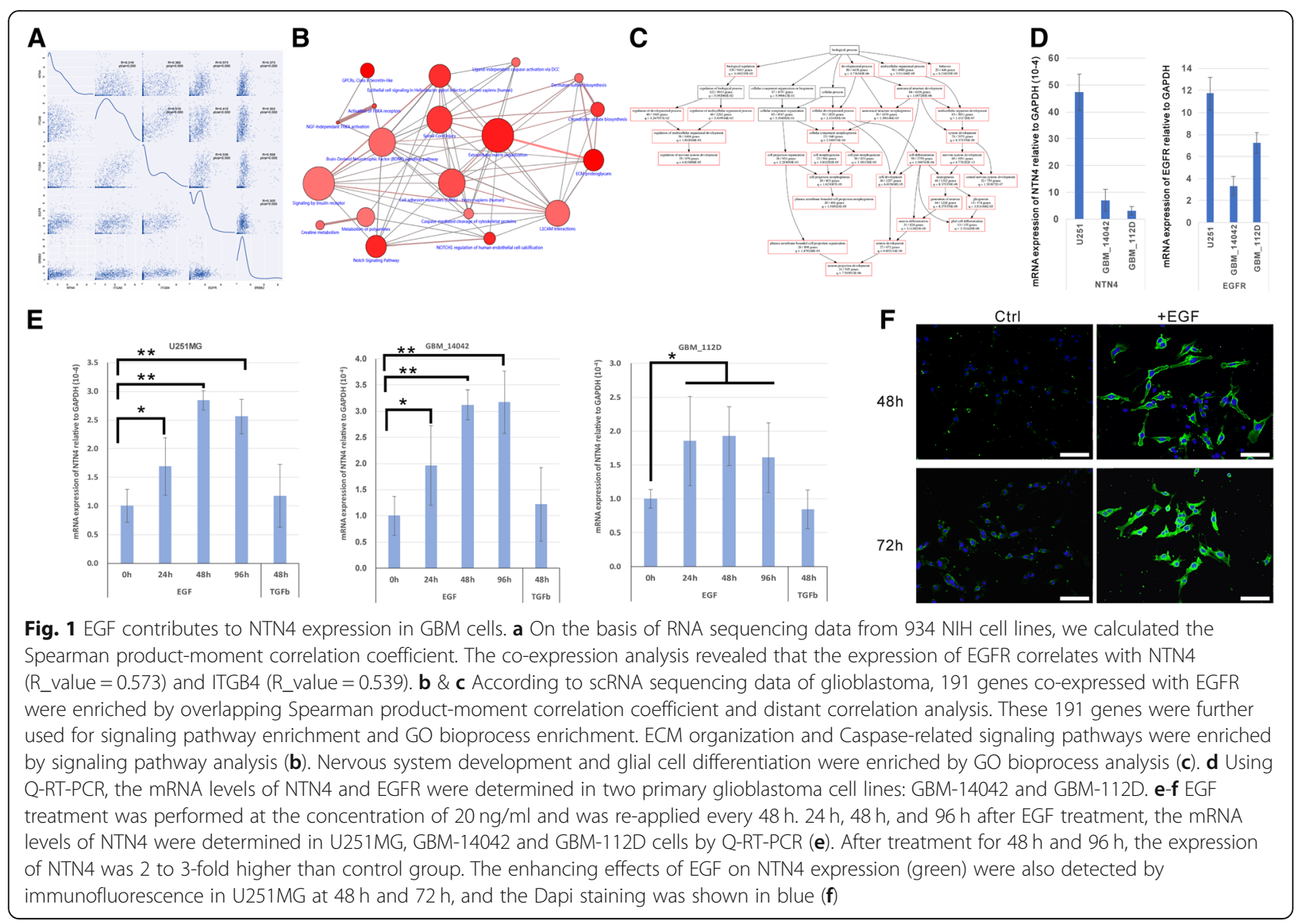

EGF stimulation upregulates the expression of NTN4. Thus, using a senescence assay induced by $\mathrm{H}_{2} \mathrm{O}_{2}$ and TMZ, we observed the relationship between EGF stimulation/NTN4 expression and GBM cell senescence.

\section{NTN4 and EGF protect GBM cells from DNA damage- induced senescence}

We have previously demonstrated that NTN4 contributes to the resistance of TMZ-triggered GBM cell senescence [13]. Hydrogen peroxide can also induce cell senescence by triggering DNA single- and double-strand breaks. Here we used a $\mathrm{H}_{2} \mathrm{O}_{2}$-induced senescence assay to further understand the effect of NTN4 and EGF on GBM cell senescence. We constructed U251MG cells expressing NTN4 (Fig. 2a). We then detected the number of senescent cells after $\mathrm{H}_{2} \mathrm{O}_{2}$ treatment using a senescence-associated beta-galactosidase assay. We observed significantly fewer senescent NTN4-overexpressing cells compared with mock control cells (Fig. 2b). Thus, NTN4 overexpression delayed U251MG cell senescence induced by $\mathrm{H}_{2} \mathrm{O}_{2}$. We then detected whether EGF could reduce the number of senescent U251MG cells induced by $\mathrm{H}_{2} \mathrm{O}_{2}$. On the 4th day after $\mathrm{H}_{2} \mathrm{O}_{2}$ treatment, we observed less senescence in U251MG cells treated with EGF compared with non-EGF treated cells. On the 7th day after $\mathrm{H}_{2} \mathrm{O}_{2}$ treatment, the number of senescent cells in the EGF treatment group was significantly less than that in the non-treatment control group. (Fig. 2c). There was a significant difference in U251MG senescence between cells treated and not treated with EGF for 7 days. With EGF $(40 \mathrm{ng} / \mathrm{ml})$ treatment, senescence of U251MG cells induced by $\mathrm{H}_{2} \mathrm{O}_{2}$ decreased. The concentration of $40 \mathrm{ng} / \mathrm{ml}$ EGF inhibited U251MG cell senescence efficiently.

To reconfirm the effect of NTN4 and EGF on GBM cell senescence induced by DNA damage, we treated U251MG cells, GBM-14042 and GBM-112D cells with TMZ to induce cell senescence. TMZ-treated cells were co-administrated NTN4, EGF, or combined NTN4/EGF, and then senescent cells were quantified. Both EGF and NTN4 treatment delayed cell senescence compared with non-treatment control cells, while the combined NTN4/ EGF treatment did not provide any additive effect on rescuing cell senescence (Fig. 2d-f). Therefore, either EGF or NTN4 can attenuate GBM cell senescence triggered by DNA damage, and they may recruit similar pathways in response to DNA damage. 


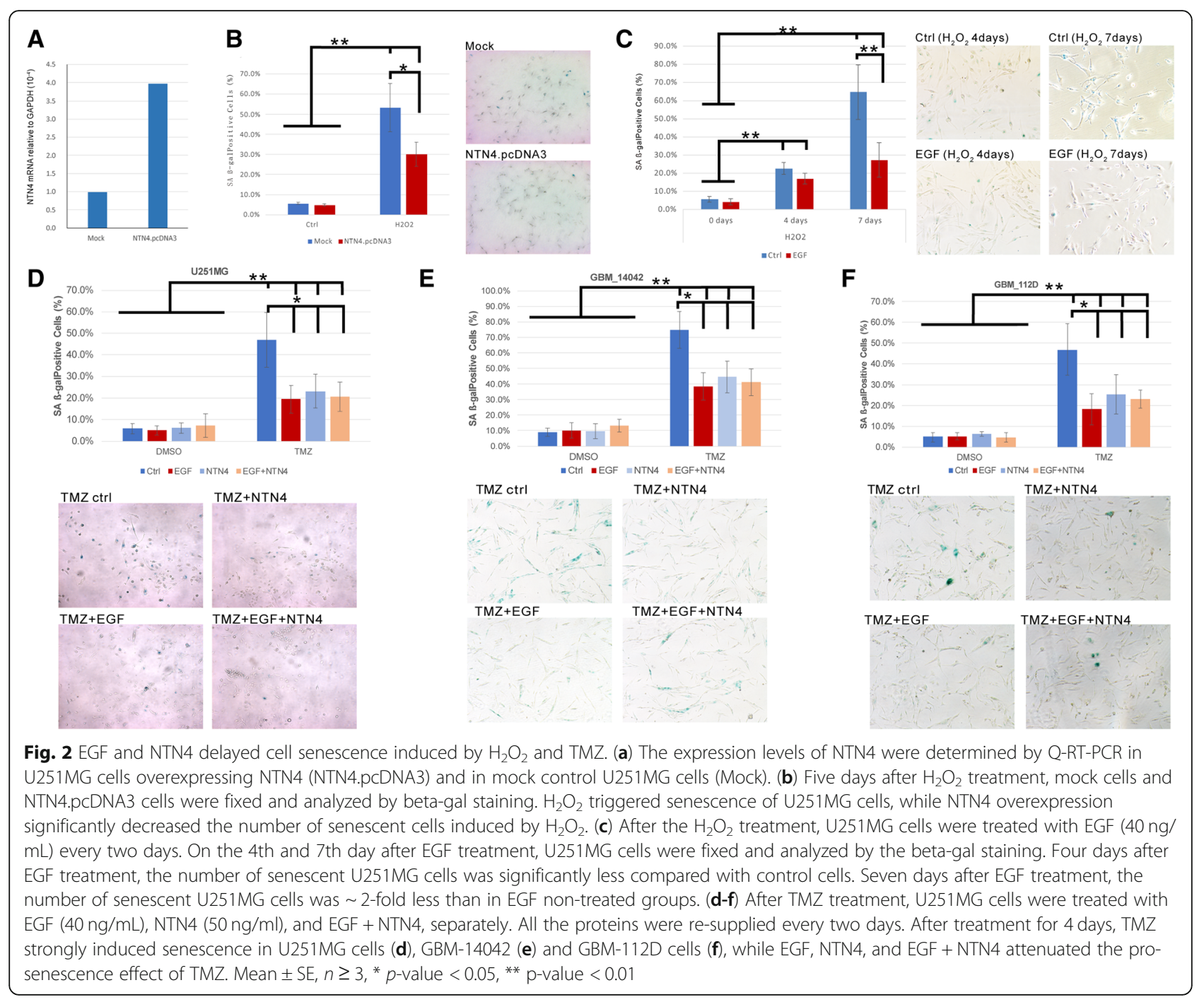

The EGF/NTN4 signaling axis contributes to the phosphorylation of Src, ERK and AKT

We then explored the combined effect of EGF and NTN4 on four major downstream signaling pathways in U251MG cells: ERK, AKT, Src and mTOR (Fig. 3). EGF treatment for $48 \mathrm{~h}$ slightly but not significantly increased p-ERK, p-AKT levels, and strongly upregulated Src phosphorylation. Short term EGF stimulation with or without NTN4 treatment for $48 \mathrm{~h}$ can enhance p-ERK, p-AKT, p-Src, and p-mTOR levels. Treatment with NTN4 for $48 \mathrm{~h}$ did not significantly alter any signaling pathways. Short term treatment of wild type U251MG cells with NTN4 increased p-ERK, p-AKT, and p-Src levels, while this effect was covered by long term stimulation with EGF. Interestingly, short-term NTN4 treatment had a continuously stimulatory effect on p-mTOR, even after $48 \mathrm{~h}$ EGF treatment. Thus, the EGF/NTN4 axis may contribute to the phosphorylation of ERK, AKT, and Src. Meanwhile, NTN4 itself may have a separate effect on mTOR phosphorylation.

\section{Co-expression of EGFR/NTN4 predicts poor survival in TMZ-treated GBM patients}

We have shown that EGF and NTN4 contribute to resistance of cell senescence induced by DNA damage. The co-expression relationship of EGFR/NTN4 prompted us to further explore the effect of EGFR/ NTN4 co-expression in GBM patients. We extracted RNA sequencing data and clinical information for 92 TMZ-treated GBM patients from The Cancer Genome Atlas Research Network (TCGA)-glioblastoma dataset [51]. To visualize the relationship among EGFR, NTN4, and patient survival, we constructed a 3D axes plot, with EGFR expression, NTN4 expression, and patient survival on the $\mathrm{X}, \mathrm{Y}$, and $\mathrm{Z}$, axes, respectively. In this plot, although EGFR and NTN4 expression did not significantly 


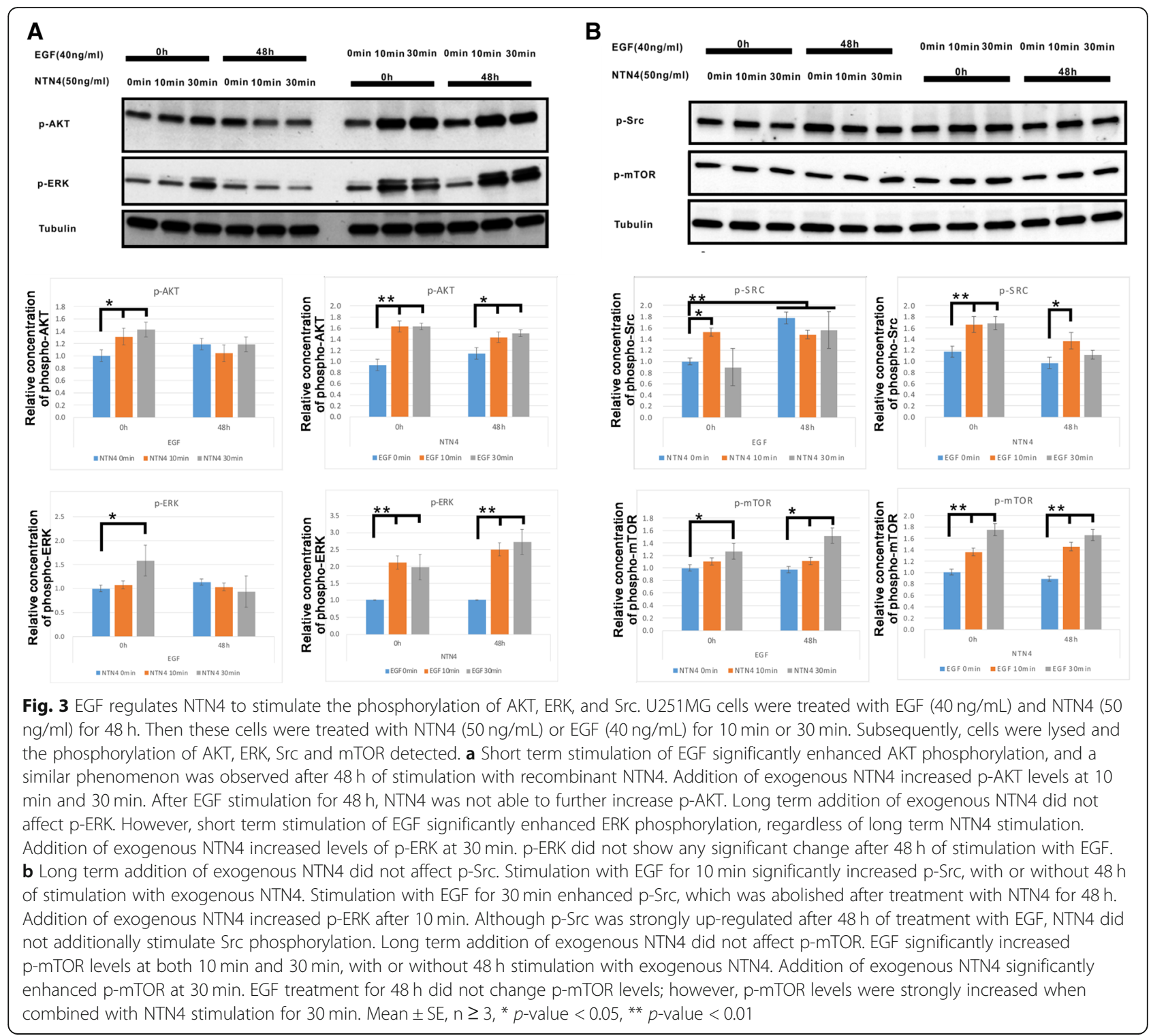

correlate with poor patient survival (Fig. 4a \& b, up-left), co-expression of EGFR and NTN4 did correlate with poor patient survival (Fig. 4c, up-left). To validate the significance of this trend, we selected the $20 \%$ of patients with either the highest or lowest expression of target genes and assessed the difference in patient survival between the two groups (Fig. 4a, b, c, right-left). KaplanMeier analysis showed that neither EGFR nor NTN4 expression significantly correlated with patient survival after TMZ treatment, while co-expression of EGFR/ NTN4 predicts poor patient survival (Fig. 4a, b, c, lower panels). To exclude a random effect of EGFR/NTN4 expression, we analyzed NTN1 and co-expression of EGFR/NTN1 as a control, and observed no correlation with patient survival. Lastly, we generated a violin plot to summarize the comparison of patient survival among all the groups mentioned above, and only the high co-expression of EGFR/NTN4 group showed a condensed low survival time (Fig. 4e).

\section{Discussion}

In this study, we report a cooperative resistance mechanism in GBM cells involving NTN4 and EGFR following DNA-damage induction. We observed the EGF/EGFR signaling pathway as an upstream regulator of NTN4. Activating mutations or amplification of EGFR are observed in $45-57.4 \%$ of glioblastomas $[52,53]$, and frequently occur in the "Classical" subgroup of glioblastomas [51, 54, 55]. Cells with EGFR gene amplification preferentially move to the infiltrating edges of glioblastoma, indicating that EGFR signaling confers a special advantage for GBM progression and migration [56]. Laminin-related integrins, 

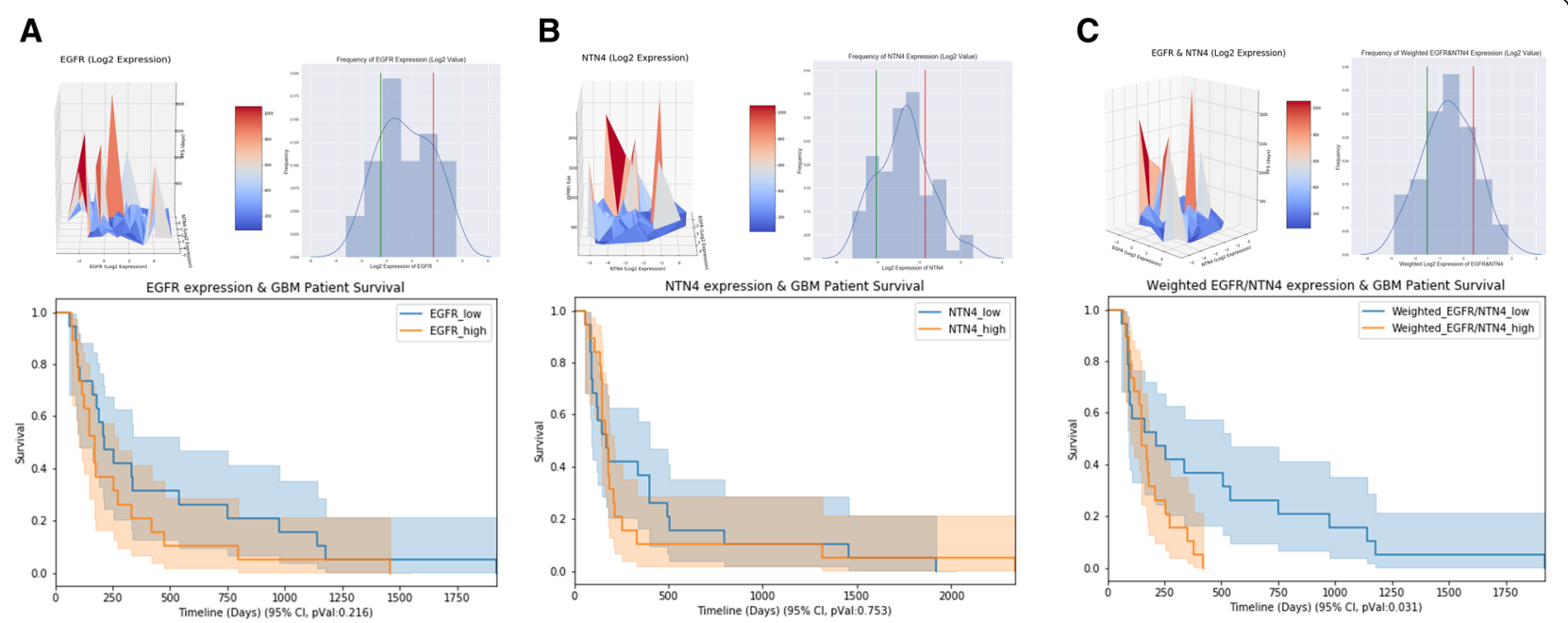

D
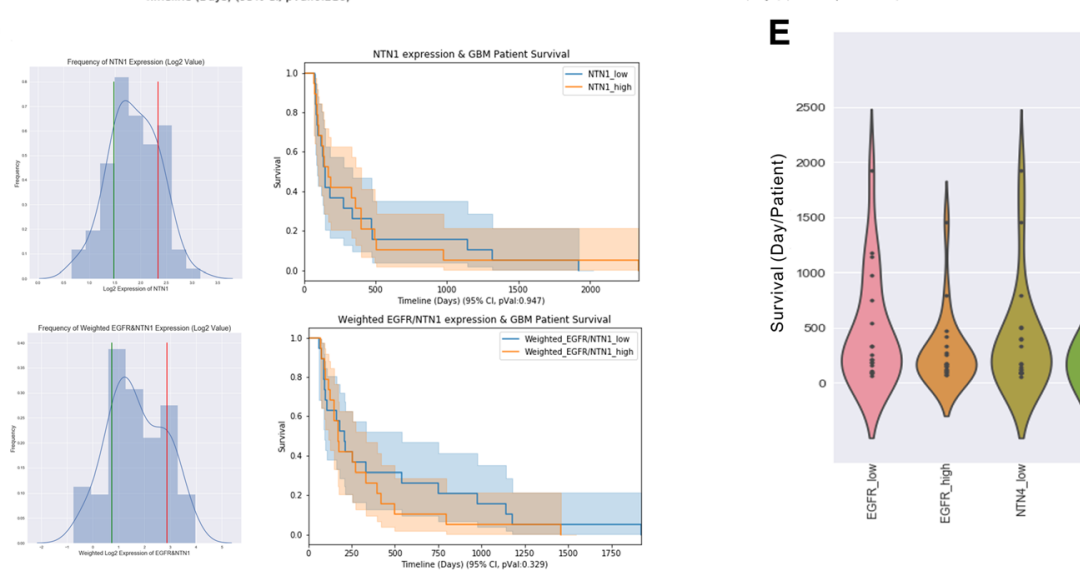

Fig. 4 The co-expression of EGFR and NTN4 contribute to TMZ resistance in GBM patients. Clinical information and gene expression data of totally 92 TMZ-treated GBM patients were extracted from the TCGA-glioblastoma dataset. To describe the contribution of EGFR and NTN4 to survival time of TMZ-treated patients, we used both 3D axes surface plots and Kaplan-Meier analysis. In 3D axes surface plots, the $X$ and $Y$ axes represent the expression of EGFR and NTN4, respectively. The Z axis represents survival time of each patient. Here, we selected the $20 \%$ of patients (top 19 patients) with either the highest (right side of red line) or lowest (left side of green line) expression of target genes and compared the survival time. a High EGFR expression slightly but not significantly predicts poor patient survival. b The expression of NTN4 itself does not correlate with patient survival. c Weighted high expression of EGFR and NTN4 significantly predicts poor patient survival. d NTN1 was used as a control in this study. Neither single NTN1 expression nor weighted expression of EGFR and NTN1 correlated with patient survival. e Violin plot representing the relationship between all different combinations of EGFR/NTN4/NTN1 expression and survival time of TMZ-treated GBM patients. Dark grey bar-box indicates a significant p-value $(<0.05)$. Only when both EGFR and NTN4 were highly expressed in patients, were survival times lowest in TMZ-treated patients

ITGA6 and ITGB4, are co-expressed with different growth factors, especially ones involved with EGF/EGFR signaling $[27-29,47]$. Interestingly, a potential integrin-associated protein, netrin-4, is also highly expressed at the invading edge of glioma. By bioinformatic analysis and the EGF stimulation experiment, we confirmed that EGF/ EGFR contribute to NTN4 expression.

Using single cell RNA sequencing data from glioblastoma, we enriched for genes co-expressed with EGFR, and further explored the GO bioprocesses and related pathways of EGFR. We found that the gene sets co-expressed with EGFR are involved in various biological functions and pathways, including cell differentiation in GBM, ECM organization, the caspase related pathway, and Notch signaling. These findings are consistent with previous studies [57-60].

Via this analysis and the experiment above, we found that EGF/EGFR regulates NTN4 expression; and that EGFR possibly modulates caspase activation via netrin/ netrin receptors $[61,62]$. Thus, EGF/EGFR signaling may regulate netrin-related pathways to protect cells from DNA damage. $\mathrm{H}_{2} \mathrm{O}_{2}$ or TMZ can trigger DNA damage in GBM cells. Using these two models, we provide in vitro evidence that either EGF or NTN4 is sufficient to attenuate GBM cell senescence induced by DNA-damage. Interestingly, in vitro co-administration of EGF and NTN4 did 
not show an additive effect on resistance to DNA damage. These results indicate that EGF/EGFR attenuate GBM cell senescence induced by DNA damage, at least partially via the expression of NTN4.

ERK and AKT pathways are essential in mediating the protective role of EGF/EGFR against DNA damage [3335]. Our results show that EGF is able to provide additional stimulation to ERK and AKT upon NTN4 treatment, suggesting that EGF may regulate the stimulatory effect of NTN4 on ERK and AKT. However, NTN4 can modulate mTOR phosphorylation independent of EGF, indicating alternative biological functions in response to NTN4.

As an oncogene, EGFR has long been proposed as a drug target for cancer treatment. Many agents were developed as small molecule tyrosine kinase inhibitors (TKIs) to interfere with EGFR tyrosine kinase activity and its downstream signaling. Although EGFR inhibitors were efficacious on GBM cells in vitro, clinical trials performed to date have been disappointing at targeting EGFR in glioblastoma. Despite the availability of TKIs for a broad spectrum of diseases, none of them demonstrate efficacy for glioblastoma treatment, either as a single agent [37] or in combination with other reagents $[63,64]$ or radiation $[65,66]$. In Phase I/II trials, EGFR inhibitors co-administered with TMZ failed to show any effects [36, 37]. Consistent with the clinical trials, our bioinformatic analysis of TCGA glioblastoma revealed that neither EGFR nor NTN4 predicts significant survival in TMZ-treated patients. This suggests that targeting either EGFR or NTN4 singly cannot improve survival of TMZ-GBM patients. However, Patients with high expression of both EGFR and NTN4 show significantly shortened survival time. This result is not in agreement with the observation that no additive effect of EGFR and NTN4 on resistance was seen in vitro; however, this could be because to two reasons. First, in addition to classical ERK/AKT resistant pathways regulated by EGF/EGFR, NTN4 may also modulate ECM/ integrin in vivo [67], which can induce ECM reorganization and mechano-transductions. Thus, after EGF/EGFR stimulation, NTN4-ECM-integrins may contribute to the resistance of DNA damage in GBM patients. Second, netrin molecules can regulate microglia, $\mathrm{T}$ cells, endothelial cells and leukocytes [68-72]. All these cells may be located near GBM cells in vivo. A potential intercellular resistance mechanism, initiated by EGF/EGFR and exerted by both EGF and NTN4, may contribute to TMZ-DNA damage resistance in GBM patients.

In summary, combinatorial approaches for targeting the EGFR/NTN4 axis and inducing DNA damage, for example, by temozolomide, may be beneficial in GBM therapy.

\section{Conclusions}

We find that $E G F / E G F R$ regulates and cooperates with NTN4 in DNA damage resistance in GBM. Therefore, this provides a potential therapeutic target to the EGFR/ NTN4 axis for GBM therapy.

\section{Abbreviations}

ECM: extracellular matrix; EGF/EGFR: Epidermal growth factor/Epidermal growth factor receptor; GBM: Glioblastoma multiforme; ITG: integrin; NTN4: Netrin-4; TMZ: Temozolomide

\section{Acknowledgements \\ The authors thank Sami Starast and Anne Remes for excellent technical assistance. Some of the microscopic analyses were carried out at the Biomedicum Imaging Unit, University of Helsinki. We thank Jeremy Allen, PhD, from Liwen Bianji, Edanz Group China (www.liwenbianji.cn/ac), for editing the English text of a draft of this manuscript. This research was supported by grants from the 57th China Postdoctoral Science Foundation, National Natural Science Foundation Of China (Lili, grant number: 81702464;Yunyun Xu, grant number: 31500718), Jiangsu Provincial Medical Youth Talent (YunyunXu, grant number: QNRC2016770), Cancer Foundation from Cancer Society of Finland, The Finnish-Norwegian Medical Foundation, Maud Kuistila Memorial Foundation, Emil Aaltosen Foundation, Orion Research Foundation, Ida Montinin Foundation, and K. Albin Johanssons Foundation.}

\section{Funding}

The 57th China Postdoctoral Science Foundation, National Natural Science Foundation Of China, Jiangsu Provincial Medical Youth Talent, Cancer Foundation from Cancer Society of Finland, The Finnish-Norwegian Medical Foundation, Maud Kuistila Memorial Foundation, Emil Aaltosen Foundation, Orion Research Foundation, Ida Montinin Foundation, and K. Albin Johanssons Foundation. The funders had no role in study design, data collection and analysis, decision to publish, or preparation of the manuscript.

\section{Availability of data and materials}

Data for the TCGA-GBMs were downloaded from TCGA Data Portal (https:// portal.gdc.cancer.gov/). Data for The Cancer Cell Line Encyclopedia and GBM single cells were downloaded from Gene Expression Omnibus (GEO accession: GSE36139 \& GSE89567).

\section{Authors' contributions}

Conceived and Designed the Study: LL QJ YZH DZ. MH JKO supervised the project. LL YLH YG TFS HL ZD YX performed the experiments. YZH provided the guidance of bioinformatics analysis. $L L Y G$ analyzed the data. Contributed reagents/materials: LL YZH JKO MH. Wrote the manuscript: LL YZH ZD. All authors read and approved the final manuscript.

Ethics approval and consent to participate

All experiments have obtained patients' consent and been approved by the Ethic Committee for Harbin Medical University (Reference Number: KY-2017-113).

\section{Consent for publication \\ Not applicable.}

\section{Competing interests}

The authors declare that no competing interests exist.

\section{Publisher's Note}

Springer Nature remains neutral with regard to jurisdictional claims in published maps and institutional affiliations.

\section{Author details}

${ }^{1}$ Department of Oncology, the Second Clinical College, Harbin Medical University, Harbin, People's Republic of China. ${ }^{2}$ Department of Neurosurgery and Brain and Nerve Research Laboratory, the First Affiliated Hospital of Soochow University, Suzhou, China. ${ }^{3}$ Institute of Pediatrics, Children's Hospital of Soochow University, Suzhou, China. ${ }^{4}$ Departments of Virology and Pathology, Faculty of Medicine, the Haartman Institute, Translational 
Cancer Biology Research Program and Helsinki University Hospital, University of Helsinki, Helsinki, Finland. ${ }^{5}$ Department of pharmacy, the Second Clinical College, Harbin Medical University, Harbin, People's Republic of China. ${ }^{6}$ Present address: Division of Molecular Neurobiology, Department of Medical Biochemistry and Biophysics, Karolinska Institute, Stockholm, Sweden.

\section{Received: 6 April 2018 Accepted: 7 November 2018}

Published online: 04 December 2018

\section{References}

1. Dunn GP, et al. Emerging insights into the molecular and cellular basis of glioblastoma. Genes Dev. 2012;26(8):756-84.

2. Louis DN. Molecular pathology of malignant gliomas. Annu Rev Pathol Mech Dis. 2006;1(1):97-117.

3. Perry JR, et al. Short-course radiation plus Temozolomide in elderly patients with glioblastoma. N Engl J Med. 2017;376(11):1027-37.

4. Stupp R, et al. Radiotherapy plus concomitant and adjuvant temozolomide for glioblastoma. N Engl J Med. 2005;352(10):987-96.

5. Gunther W, et al. Temozolomide induces apoptosis and senescence in glioma cells cultured as multicellular spheroids. Br J Cancer. 2003;88(3): 463-9.

6. Bent MJvd, et al. MGMT promoter methylation is prognostic but not predictive for outcome to adjuvant PCV chemotherapy in anaplastic Oligodendroglial tumors: a report from EORTC brain tumor group study 26951. J Clin Oncol. 2009;27(35):5881-6.

7. Gorlia T, et al. Nomograms for predicting survival of patients with newly diagnosed glioblastoma: prognostic factor analysis of EORTC and NCIC trial 26981-22981/CE.3. Lancet Oncol. 2008;9(1):29-38.

8. Hegi ME, et al. MGMT gene silencing and benefit from temozolomide in glioblastoma. N Engl J Med. 2005;352(10):997-1003.

9. Kohsaka S, et al. STAT3 inhibition overcomes Temozolomide resistance in glioblastoma by downregulating MGMT expression. Mol Cancer Ther. 2012; 11(6):1289-99.

10. Sinnberg T, et al. Inhibition of PI3K-AKT-mTOR signaling sensitizes melanoma cells to cisplatin and temozolomide. J Invest Dermatol. 2009; 129(6):1500-15.

11. Wang $\mathrm{H}-\mathrm{B}$, et al. Focal adhesion kinase is involved in mechanosensing during fibroblast migration. Proc Natl Acad Sci. 2001;98(20):11295-300.

12. Klemm F, Joyce JA. Microenvironmental regulation of therapeutic response in cancer. Trends Cell Biol. 2015;25(4):198-213.

13. Li L, et al. NETRIN-4 protects glioblastoma cells FROM temozolomide induced senescence. PLoS One. 2013;8(11):e80363.

14. Koch $M$, et al. A novel member of the netrin family, beta-netrin, shares homology with the beta chain of laminin: identification, expression, and functional characterization. J Cell Biol. 2000;151(2):221-34.

15. Yin $Y$, Sanes JR, Miner JH. Identification and expression of mouse netrin-4. Mech Dev. 2000;96(1):115-9.

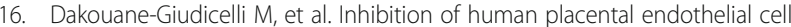
proliferation and angiogenesis by netrin-4. Placenta. 2015;36(11):1260-5.

17. Enoki $Y$, et al. Netrin-4 promotes differentiation and migration of osteoblasts. In Vivo. 2017;31(5):793-9.

18. Eveno C, et al. Netrin-4 delays colorectal cancer carcinomatosis by inhibiting tumor angiogenesis. Am J Pathol. 2011;178(4):1861-9.

19. Han $Y$, et al. Therapeutic effects of topical Netrin-4 inhibits corneal neovascularization in alkali-burn rats. PLoS One. 2015;10(4):e0122951.

20. Lejmi $E$, et al. Netrin-4 inhibits angiogenesis via binding to neogenin and recruitment of Unc5B. Proc Natl Acad Sci U S A. 2008;105(34):12491-6.

21. LV B, et al. Netrin-4 as a biomarker promotes cell proliferation and invasion in gastric cancer. Oncotarget. 2015;6(12):9794-806.

22. Villanueva AA, et al. The Netrin-4/ Neogenin-1 axis promotes neuroblastoma cell survival and migration. Oncotarget. 2017;8(6):9767-82.

23. $\mathrm{Xu}$ X, et al. NTN4 is associated with breast cancer metastasis via regulation of EMT-related biomarkers. Oncol Rep. 2017;37(1):449-57.

24. Hoelzinger DB, et al. Gene expression profile of glioblastoma multiforme invasive phenotype points to new therapeutic targets. Neoplasia. 2005;7(1):7-16.

25. Hu Y, et al. Netrin-4 promotes glioblastoma cell proliferation through integrin beta4 signaling. Neoplasia. 2012;14(3):219-27.

26. Mainiero MB, et al. Stereotaxic core needle biopsy of breast microcalcifications: correlation of target accuracy and diagnosis with lesion size. Radiology. 1996;198(3):665-9.
27. Seano G, et al. Endothelial podosome rosettes regulate vascular branching in tumour angiogenesis. Nat Cell Biol. 2014;16(10):931-41-1-8.

28. Shema $\mathrm{E}$, et al. The histone H2B-specific ubiquitin ligase RNF20/hBRE1 acts as a putative tumor suppressor through selective regulation of gene expression. Genes Dev. 2008;22(19):2664-76.

29. Stewart $\mathrm{RL}$, et al. Elevated integrin a6ß4 expression is associated with venous invasion and decreased overall survival in non-small cell lung cancer. Hum Pathol. 2016;54:174-83.

30. Normanno N, et al. Epidermal growth factor receptor (EGFR) signaling in cancer. Gene. 2006;366(1):2-16.

31. Oda K, et al. A comprehensive pathway map of epidermal growth factor receptor signaling. Mol Syst Biol. 2005;1:2005 0010.

32. Seshacharyulu $P$, et al. Targeting the EGFR signaling pathway in cancer therapy. Expert Opin Ther Targets. 2012;16(1):15-31.

33. Kriegs $\mathrm{M}$, et al. The epidermal growth factor receptor modulates DNA double-strand break repair by regulating non-homologous end-joining. DNA Repair. 2010;9(8):889-97.

34. Munoz $J$, et al. Temozolomide induces the production of epidermal growth factor to regulate MDR1 expression in glioblastoma cells. Mol Cancer Ther. 2014;13(10):2399-411.

35. Toulany $M$, et al. Blockage of epidermal growth factor receptorphosphatidylinositol 3-kinase-AKT signaling increases radiosensitivity of KRAS mutated human tumor cells in vitro by affecting DNA repair. Clin Cancer Res. 2006;12(13):4119-26.

36. Peereboom DM, et al. Phase II trial of erlotinib with temozolomide and radiation in patients with newly diagnosed glioblastoma multiforme. J Neuro-Oncol. 2010;98(1):93-9.

37. Reardon DA, et al. Phase $1 /$ randomized phase II study of afatinib, an irreversible ErbB family blocker, with or without protracted temozolomide in adults with recurrent glioblastoma. Neuro-Oncology. 2015;17(3):430-9.

38. Ylivinkka I, et al. Motility of glioblastoma cells is driven by netrin-1 induced gain of stemness. J Exp Clin Cancer Res. 2017;36(1):9.

39. Zhou K, et al. Knockdown of long non-coding RNA NEAT1 inhibits glioma cell migration and invasion via modulation of SOX2 targeted by miR-132. Mol Cancer. 2018;17(1):105

40. Debacq-Chainiaux F, et al. Protocols to detect senescence-associated betagalactosidase (SA-betagal) activity, a biomarker of senescent cells in culture and in vivo. Nat Protoc. 2009;4(12):1798-806.

41. Guo $X$, et al. Inferring nonlinear gene regulatory networks from gene expression data based on distance correlation. PLoS One. 2014;9(2): e87446.

42. Székely GJ, Rizzo ML. Brownian distance covariance. The Annals of Applied Statistics. 2009;3(4):1236-65.

43. Székely GJ, Rizzo ML, Bakirov NK. Measuring and testing dependence by correlation of distances. Ann Stat. 2007:35(6):2769-94.

44. Alper $\mathrm{O}$, et al. Anti-sense suppression of epidermal growth factor receptor expression alters cellular proliferation, cell-adhesion and tumorigenicity in ovarian cancer cells. Int J Cancer. 2000;88(4):566-74.

45. Green MR, et al. Biochemical and ultrastructural processing of [1251]epidermal growth factor in rat epidermis and hair follicles: accumulation of nuclear label. J Invest Dermatol. 1987;88(3):259-65.

46. Kajiji S, Tamura RN, Quaranta V. A novel integrin (alpha E beta 4) from human epithelial cells suggests a fourth family of integrin adhesion receptors. EMBO J. 1989;8(3):673-80.

47. Mainiero F, et al. The intracellular functions of alpha6beta4 integrin are regulated by EGF. J Cell Biol. 1996;134(1):241-53.

48. Barretina J, et al. The Cancer cell line encyclopedia enables predictive modelling of anticancer drug sensitivity. Nature. 2012;483(7391):603-7.

49. Venteicher AS, et al. Decoupling genetics, lineages, and microenvironment in IDH-mutant gliomas by single-cell RNA-seq. Science. 2017;355(6332): eaai8478.

50. Wichmann $\mathrm{H}$, et al. Targeting of EGFR and HER2 with therapeutic antibodies and siRNA: a comparative study in glioblastoma cells. Strahlenther Onkol. 2015;191(2):180-91.

51. Verhaak RG, et al. Integrated genomic analysis identifies clinically relevant subtypes of glioblastoma characterized by abnormalities in PDGFRA, IDH1, EGFR, and NF1. Cancer Cell. 2010;17(1):98-110.

52. Brennan CW, et al. The somatic genomic landscape of glioblastoma. Cell. 2013;155(2):462-77.

53. TCGAnetwork. Comprehensive genomic characterization defines human glioblastoma genes and core pathways. Nature. 2008;455(7216):1061-8. 
54. Libermann TA, et al. Amplification, enhanced expression and possible rearrangement of EGF receptor gene in primary human brain tumours of glial origin. Nature. 1985;313(5998):144-7.

55. Ohgaki H, et al. Genetic pathways to glioblastoma: a population-based study. Cancer Res. 2004;64(19):6892-9.

56. Lal A, et al. Mutant epidermal growth factor receptor up-regulates molecular effectors of tumor invasion. Cancer Res. 2002;62(12):3335-9.

57. Aguirre A, Rubio ME, Gallo V. Notch and EGFR pathway interaction regulates neural stem cell number and self-renewal. Nature. 2010;467(7313):323-7.

58. Allan LA, et al. Inhibition of caspase-9 through phosphorylation at Thr 125 by ERK MAPK. Nat Cell Biol. 2003:5(7):647-54.

59. Henson ES, Gibson SB. Surviving cell death through epidermal growth factor (EGF) signal transduction pathways: implications for cancer therapy. Cell Signal. 2006;18(12):2089-97.

60. Mariotti A, et al. EGF-R signaling through Fyn kinase disrupts the function of integrin alpha6beta4 at hemidesmosomes: role in epithelial cell migration and carcinoma invasion. J Cell Biol. 2001;155(3):447-58.

61. Liu J, et al. Mediation of the DCC apoptotic signal by DIP13 alpha. J Biol Chem. 2002;277(29):26281-5

62. Tanikawa C, et al. p53RDL1 regulates p53-dependent apoptosis. Nat Cell Biol. 2003;5(3):216-23.

63. Raizer JJ, et al. A phase II trial of erlotinib in patients with recurrent malignant gliomas and nonprogressive glioblastoma multiforme postradiation therapy. Neuro-Oncology. 2010;12(1):95-103.

64. Wen PY, et al. Phase I/II study of erlotinib and temsirolimus for patients with recurrent malignant gliomas: north American brain tumor consortium trial 04-02. Neuro-Oncology. 2014;16(4):567-78.

65. Chakravarti A, et al. RTOG 0211: a phase 1/2 study of radiation therapy with concurrent gefitinib for newly diagnosed glioblastoma patients. Int J Radiat Oncol Biol Phys. 2013;85(5):1206-11.

66. Raizer JJ, et al. A phase II study of bevacizumab and erlotinib after radiation and temozolomide in MGMT unmethylated GBM patients. J Neuro-Oncol. 2016;126(1):185-92.

67. Schneiders $\mathrm{Fl}$, et al. Binding of netrin- 4 to laminin short arms regulates basement membrane assembly. J Biol Chem. 2007;282(33):23750-8.

68. Boneschansker $L$, et al. Netrin-1 Augments Chemokinesis in CD4+ T Cells In Vitro and Elicits a Proinflammatory Response In Vivo. J Immunology (Baltimore, Md: 1950). 2016;197(4):1389-98.

69. Larrieu-Lahargue F, et al. Netrin-4 activates endothelial integrin \{alpha\}6\{beta\}1. Circ Res. 2011;109(7):770-4.

70. Lejmi E, et al. Netrin-4 promotes mural cell adhesion and recruitment to endothelial cells. Vasc Cell. 2014;6(1):1.

71. Ly NP, et al. Netrin-1 inhibits leukocyte migration in vitro and in vivo. Proc Natl Acad Sci U S A. 2005:102(41):14729-34.

72. Podjaski $C$, et al. Netrin 1 regulates blood-brain barrier function and neuroinflammation. Brain. 2015;138(Pt 6):1598-612.

Ready to submit your research? Choose BMC and benefit from:

- fast, convenient online submission

- thorough peer review by experienced researchers in your field

- rapid publication on acceptance

- support for research data, including large and complex data types

- gold Open Access which fosters wider collaboration and increased citations

- maximum visibility for your research: over $100 \mathrm{M}$ website views per year

At $\mathrm{BMC}$, research is always in progress.

Learn more biomedcentral.com/submissions 